\title{
NATURALITY OF SYMMETRIC IMPRIMITIVITY THEOREMS
}

\author{
ASTRID AN HUEF, S. KALISZEWSKI, IAIN RAEBURN, AND DANA P. WILLIAMS
}

(Communicated by Marius Junge)

\begin{abstract}
The first imprimitivity theorems identified the representations of groups or dynamical systems which are induced from representations of a subgroup. Symmetric imprimitivity theorems identify pairs of crossed products by different groups which are Morita equivalent and hence have the same representation theory. Here we consider commuting actions of groups $H$ and $K$ on a $C^{*}$-algebra which are saturated and proper as defined by Rieffel in 1990. Our main result says that the resulting Morita equivalence of crossed products is natural in the sense that it is compatible with homomorphisms and induction processes.
\end{abstract}

\section{INTRODUCTION}

Suppose that a locally compact group $G$ acts freely and properly on the right of a locally compact space $T$, and rt is the induced action on $C_{0}(T)$. Green proved in [4] that the crossed product $C_{0}(T) \rtimes_{\mathrm{rt}} G$ is Morita equivalent to $C_{0}(T / G)$. Raeburn and Williams considered diagonal actions $\operatorname{rt} \otimes \alpha$ on $G$ on $C_{0}(T, B)=C_{0}(T) \otimes B$, and showed in 18, that $C_{0}(T, B) \rtimes_{\mathrm{rt} \otimes \alpha} G$ is Morita equivalent to the induced algebra $\operatorname{Ind}_{G}^{T}(B, \alpha)$. Motivated by the idea that $C_{0}(T / G)$ and $\operatorname{Ind}_{G}^{T}(B, \alpha)$ are playing the role of a fixed-point algebra for the action of $G$, Rieffel studied a family of proper actions $(A, \alpha)$ for which there is a generalized fixed-point algebra $A^{\alpha}$ in $M(A)[21$. Rieffel proved in particular that if $\alpha$ is an action of $G$ on a $C^{*}$-algebra $A$ and if $\phi: C_{0}(T) \rightarrow M(A)$ is an equivariant nondegenerate homomorphism, then the reduced crossed product $A \rtimes_{\alpha, r} G$ is Morita equivalent to $A^{\alpha}$ (see [22, Theorem 5.7] and [21, Corollary 1.7]); taking $A=C_{0}(T, B)$ gives the result in [18.

Rieffel's construction of $A^{\alpha}$ starts from a dense subalgebra $A_{0}$ of $A$ with properties such as those of $C_{c}(T)$ in $C_{0}(T)$. While in practice there always seems to be an obvious candidate for $A_{0}$ which gives the "right answer", $A^{\alpha}$ does ostensibly depend on the choice of $A_{0}$, and several authors have tried in vain to find a canonical choice [3, 22. Alternatively, as in [10, we can try to say upfront what "right" means, and prove that Rieffel's construction has these properties. The idea is, loosely, that constructions should be functorial and isomorphisms, such as those implemented by Morita equivalences, should be natural. This program has already had some significant applications, especially in nonabelian duality for crossed products of $C^{*}$-algebras [10, 7].

Received by the editors March 18, 2011 and, in revised form, October 10, 2011.

2010 Mathematics Subject Classification. Primary 46L55.

Key words and phrases. Symmetric imprimitivity theorem, proper actions on $C^{*}$-algebras, naturality, fixed-point algebras, crossed products.

This research was supported by the University of Otago and the Edward Shapiro Fund at Dartmouth College. 
In [6], we showed that the assignments $(A, \alpha, \phi) \mapsto A \rtimes_{\alpha, r} G$ and $(A, \alpha, \phi) \mapsto$ $A^{\alpha}$ can be extended to functors RCP and Fix between certain categories whose morphisms are derived from right-Hilbert bimodules, and whose isomorphisms are given by Morita equivalences. The main result of [ 6 ] says that Rieffel's Morita equivalences give a natural isomorphism between RCP and Fix.

All these Morita equivalences have symmetric versions. For Green's theorem, the symmetric version involves commuting free and proper actions of two groups $H$ and $K$ on the same space $T$, and says that $C_{0}(T / H) \rtimes K$ is Morita equivalent to $C_{0}(T / K) \rtimes H$ (this is marginally more general than the version proved in [20]). If in addition $\alpha$ and $\beta$ are commuting actions of $H$ and $K$ on a $C^{*}$-algebra $B$, then the symmetric imprimitivity theorem of [11, 17] gives a Morita equivalence between crossed products $\operatorname{Ind}_{H}^{T}(B, \alpha) \rtimes \frac{}{\operatorname{rt} \otimes \beta} K$ and $\operatorname{Ind}_{K}^{T}(B, \beta) \rtimes \overline{\operatorname{rt} \otimes \alpha} H$. Work of Quigg and Spielberg [16] implies that there is a similar Morita equivalence for the reduced crossed products. In [9, Corollary 3.8], we found a symmetric version of Rieffel's equivalence for a pair of commuting proper actions on a $C^{*}$-algebra $A$, and then recovered the Quigg-Spielberg theorem by taking $A=C_{0}(T, B)$ (see [9, §4]). Symmetric imprimitivity theorems have found significant applications (see [12, 1], for example), and there are analogues for groupoids [13, 19], for graph algebras [15], and for Fell bundles [14].

Here we consider commuting free and proper actions of $H$ and $K$ on $T$, and form a category whose objects $(A, \sigma, \tau, \phi)$ consist of commuting actions $\sigma: H \rightarrow$ Aut $A$ and $\tau: K \rightarrow$ Aut $A$, and an equivariant nondegenerate homomorphism $\phi: C_{0}(T) \rightarrow M(A)$. We show that there are functors Fix $_{H}$ and $\mathrm{Fix}_{K}$ based on the assignments $(A, \sigma, \tau, \phi) \mapsto\left(A^{\sigma}, \bar{\tau}\right)$ and $(A, \sigma, \tau, \phi) \mapsto\left(A^{\tau}, \bar{\sigma}\right)$, and prove that Rieffel's bimodules $X(A, \sigma, \tau, \phi)$ from [9, Corollary 3.8] give a natural isomorphism between the functors $\mathrm{RCP} \circ \mathrm{Fix}_{H}$ and $\mathrm{RCP} \circ \mathrm{Fix}_{K}$. This is interesting even in the situation of [17, where it gives the naturality of Quigg and Spielberg's symmetric imprimitivity theorem, and in the one-sided case, where it yields naturality of the Morita equivalence of [18].

\section{Preliminaries}

Throughout this paper, $G, H$, and $K$ are locally compact groups, all of which act on the right of a locally compact space $T$. The actions of $H$ and $K$ are always assumed to commute. We denote by rt the action of any of them on $C_{0}(T)$ by right translation: $\operatorname{rt}_{g}(f)(t)=f(t \cdot g)$

We use the same categories and notation as in [6]. In particular, $C^{*}$ is the category whose objects are $C^{*}$-algebras and whose morphisms are isomorphism classes of right-Hilbert bimodules. In the category $\mathrm{C}^{*}$ act $(G)$, the objects $(A, \alpha)$ consist of an action $\alpha$ of $G$ on a $C^{*}$-algebra $A$, and the morphisms $[X, u]:(A, \alpha) \rightarrow(B, \beta)$ are isomorphism classes of right-Hilbert $A-B$ bimodules $X$ with an $\alpha-\beta$ compatible action $u$ of $G$.

In the semi-comma category $\mathrm{C}^{*}$ act $\left(G,\left(C_{0}(T), \mathrm{rt}\right)\right)$, the objects are triples $(A, \alpha, \phi)$, where $(A, \alpha)$ is an object in $\mathrm{C}^{*}$ act $(G)$ and $\phi: C_{0}(T) \rightarrow M(A)$ is an rt $-\alpha$ equivariant nondegenerate homomorphism. The morphisms from $(A, \alpha, \phi)$ to $(B, \beta, \psi)$ are the same as the morphisms from $(A, \alpha)$ to $(B, \beta)$ in $\mathrm{C}^{*} \operatorname{act}(G) !$

\footnotetext{
${ }^{1}$ In $[6$, we were only interested in free and proper actions of $G$, but there is no reason to make this restriction when defining the semi-comma category $\mathrm{C}^{*} \operatorname{act}\left(G,\left(C_{0}(T), \mathrm{rt}\right)\right)$.
} 
Commuting actions $\sigma, \tau$ of $H, K$ (on spaces, $C^{*}$-algebras or Hilbert modules) are essentially the same as actions $\sigma \times \tau$ of $G=H \times K$, and hence we can apply the construction of the previous paragraph with $G=H \times K$. However, to emphasize the symmetry of our situation, we view the comma category as a category $\mathrm{C}^{*}$ act $\left(H, K,\left(C_{0}(T), \mathrm{rt}\right)\right)$ in which the objects are quadruples $(A, \sigma, \tau, \phi)$ such that $(A, \sigma \times \tau, \phi)$ is an object of the semi-comma category $\mathrm{C}^{*}$ act $\left(H \times K,\left(C_{0}(T)\right.\right.$, rt $\left.)\right)$; the morphisms from $(A, \sigma, \tau, \phi)$ to $(B, \mu, \nu, \psi)$ are then triples $[X, u, v]$ such that $[X, u \times$ $v]$ is a morphism from $(A, \sigma \times \tau, \phi)$ to $(B, \mu \times \nu, \psi)$ in $\mathrm{C}^{*}$ act $\left(H \times K,\left(C_{0}(T), \mathrm{rt}\right)\right)$.

Remark 2.1. We stress that when we assume that $H$ and $K$ act freely and properly on $T$, we are not assuming that $H \times K$ acts freely and properly, because then we would lose the main applications of the symmetric imprimitivity theorem. For example, let $T=\mathbb{R}, H=\mathbb{Z}$ and $K=\mathbb{Z}$, take an irrational number $\theta$, and define $r \cdot h:=r+h \theta$ and $r \cdot k=r+k$ for $r \in T, h \in H, k \in K$; then the symmetric imprimitivity theorem implies that the irrational rotation algebra $A_{\theta}$ is Morita equivalent to $A_{\theta^{-1}}$.

\section{DeFining The FUnCTORS}

In this section we define functors on $\mathrm{C}^{*}$ act $\left(H, K,\left(C_{0}(T), \mathrm{rt}\right)\right)$ analogous to RCP and Fix from [6], but which only deal with the $H$ - or $K$-part of the action and which take values in an equivariant category.

Proposition 3.1. The assignments

$$
(A, \sigma, \tau, \phi) \mapsto\left(A \rtimes_{\sigma, r} H, \tau \rtimes \operatorname{id}_{H}\right) \quad \text { and } \quad[X, u, v] \mapsto\left[X \rtimes_{u, r} H, v \rtimes \operatorname{id}_{H}\right]
$$

define a functor $\mathrm{RCP}_{H}$ from $\mathrm{C}^{*} \operatorname{act}\left(H, K,\left(C_{0}(T), \mathrm{rt}\right)\right)$ to $\mathrm{C}^{*}$ act $(K)$, where $\tau \rtimes \mathrm{id}_{H}$ is the action of $K$ given by $\left(\tau \rtimes \mathrm{id}_{H}\right)_{k}(f)(h)=\tau_{k}(f(h))$ for $f \in C_{c}(H, A)$ (and similarly for $v \rtimes \mathrm{id}_{H}$ ).

Proof. Let $\mathrm{F}: \mathrm{C}^{*}$ act $(H \times K) \rightarrow \mathrm{C}^{*}$ act $(H \times K)$ be the subgroup-crossed-product functor from [2, Theorem 3.24], applied to the normal subgroup $H \subset H \times K$. With $\alpha^{\text {dec }}$ and $w^{\text {dec }}$ the "decomposition actions" of $H \times K$ as defined in [2, Section 3.3.1], $\mathrm{F}$ is given by

$$
(A, \alpha) \mapsto\left(A \rtimes_{\alpha \mid, r} H, \alpha^{\mathrm{dec}}\right) \quad \text { and } \quad[X, w] \mapsto\left[X \rtimes_{w \mid, r} H, w^{\mathrm{dec}}\right] .
$$

With $\alpha=\sigma \times \tau$, for $k \in K$ and $f \in C_{c}(H, A)$ we have

$$
\alpha_{(e, k)}^{\mathrm{dec}}(f)(h)=\Delta_{H}(e) \sigma_{e}\left(\tau_{k}\left(f\left(e^{-1} h e\right)\right)\right)=\tau_{k}(f(h))=\left(\tau \rtimes \operatorname{id}_{H}\right)_{k}(f)(h),
$$

and similarly, if $w=u \times v$, then $w_{(e, k)}^{\text {dec }}=\left(v \rtimes \operatorname{id}_{H}\right)_{k}$. So if we let Res : $\mathrm{C}^{*} \operatorname{act}(H \times$ $K) \rightarrow \mathrm{C}^{*} \operatorname{act}(K)$ be the restriction functor from [2, Corollary 3.17] applied to the subgroup $K \subset H \times K$, we see that the composition Res $\circ \mathrm{F}$ is given by

$$
(A, \sigma \times \tau) \mapsto\left(A \rtimes_{\sigma, r} H, \tau \rtimes \operatorname{id}_{H}\right) \quad \text { and } \quad[X, u \times v] \mapsto\left[X \rtimes_{u, r} H, v \rtimes \operatorname{id}_{H}\right] .
$$

Defining $\mathrm{RCP}_{H}$ to be the composition of Res $\circ \mathrm{F}$ with the forgetful functor from $\mathrm{C}^{*} \operatorname{act}\left(H \times K,\left(C_{0}(T), \mathrm{rt}\right)\right)$ to $\mathrm{C}^{*}$ act $(H \times K)$ which takes $(A, \alpha, \phi)$ to $(A, \alpha)$ gives the result.

For the next result, we observe that the proof of [7, Proposition 4.1] requires only that the normal subgroup $N \subset G$ act freely and properly; the basic factorization result in [6, Corollary 2.3], which is invoked in the proof, applies in the semi-comma category for arbitrary actions of $G$ on $T$. So if $H$ acts freely and properly on $T$, we 
can apply [7, Proposition 4.1] with $G=H \times K, N=H$ and $G / N=K$. This gives a fixed-point functor $\operatorname{Fix}_{H}^{K}$ from $C^{*} \operatorname{act}\left(H, K,\left(C_{0}(T), \mathrm{rt}\right)\right)$ to $\mathrm{C}^{*} \operatorname{act}\left(K,\left(C_{0}(T / H), \mathrm{rt}\right)\right)$ with object and morphism maps

$$
(A, \sigma \times \tau, \phi) \mapsto\left(A^{\sigma},(\sigma \times \tau)^{K}, \phi_{H}\right) \quad \text { and } \quad[X, u \times v] \mapsto\left[\operatorname{Fix}(X, u),(u \times v)^{K}\right] .
$$

Now we introduce the notation

$$
\bar{\tau}=(\sigma \times \tau)^{K}, \quad X^{u}=\operatorname{Fix}(X, u), \quad \text { and } \quad \bar{v}=(u \times v)^{K},
$$

and define $\mathrm{Fix}_{H}$ to be the composition of $\mathrm{Fix}_{H}^{K}$ with the forgetful functor from $\mathrm{C}^{*} \operatorname{act}\left(K,\left(C_{0}(T / H), \mathrm{rt}\right)\right)$ to $\mathrm{C}^{*} \operatorname{act}(K)$. Then we have:

Proposition 3.2. Suppose that the action of $H$ on $T$ is free and proper. Then the assignments

$$
(A, \sigma, \tau, \phi) \mapsto\left(A^{\sigma}, \bar{\tau}\right) \quad \text { and } \quad[X, u, v] \mapsto\left[X^{u}, \bar{v}\right]
$$

define a functor $\mathrm{Fix}_{H}$ from $\mathrm{C}^{*}$ act $\left(H, K,\left(C_{0}(T), \mathrm{rt}\right)\right)$ to $\mathrm{C}^{*} \operatorname{act}(K)$.

\section{NATURALity}

Suppose that the actions of $H$ and $K$ on $T$ are free and proper. Then for each object $(A, \sigma, \tau, \phi)$ of $\mathrm{C}^{*}$ act $\left(H, K,\left(C_{0}(T), \mathrm{rt}\right)\right)$, the hypotheses of [9, Theorem 4.4] are satisfied; therefore $A_{0}:=\operatorname{span}\left\{\phi(f) a \phi(g) \mid f, g \in C_{c}(T), a \in A\right\}$ can be completed to give an $A^{\sigma} \rtimes_{\bar{\tau}, r} K-A^{\tau} \rtimes_{\bar{\sigma}, r} H$ imprimitivity bimodule, which we will denote here by $X(A, \sigma, \tau, \phi)$. The isomorphism class $[X(A, \sigma, \tau, \phi)]$ is an isomorphism in the category $\mathrm{C}^{*}$ from $A^{\sigma} \rtimes_{\bar{\tau}, r} K=\operatorname{RCP} \circ \operatorname{Fix}_{H}(A, \sigma, \tau, \phi)$ to $A^{\tau} \rtimes_{\bar{\sigma}, r} H=\mathrm{RCP} \circ \operatorname{Fix}_{K}(A, \sigma, \tau, \phi)$.

Theorem 4.1. Suppose that $H$ and $K$ act freely and properly on $T$. Then

$$
(A, \sigma, \tau, \phi) \mapsto[X(A, \sigma, \tau, \phi)]
$$

is a natural isomorphism between the functors $\mathrm{RCP} \circ \mathrm{Fix}_{H}$ and $\mathrm{RCP} \circ \mathrm{Fix}_{K}$ from $\mathrm{C}^{*} \operatorname{act}\left(H, K,\left(C_{0}(T), \mathrm{rt}\right)\right)$ to $\mathrm{C}^{*}$.

Our strategy for proving Theorem 4.1 is as follows. First, we present a natural isomorphism between the functors $\mathrm{Fix}_{H}$ and $\mathrm{RCP}_{H}$ defined in Section 3 . This is done in Proposition 4.2, which is an equivariant version of [6, Theorem 3.5]. Composing with RCP gives a natural isomorphism between RCP $\circ \mathrm{Fix}_{H}$ and $\mathrm{RCP} \circ \mathrm{RCP}_{H}$ (Corollary 4.4). Since the iterated crossed-product functors $\mathrm{RCP} \circ \mathrm{RCP}_{H}$ and $\mathrm{RCP} \circ \mathrm{RCP}_{K}$ are easily seen to be naturally isomorphic, we obtain a natural isomorphism by composition:

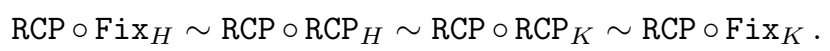

The last step of the proof is to identify the bimodules underlying this composition with $X(A, \sigma, \tau, \phi)$; this requires the reworking of [9, Theorem 4.4] in Proposition 4.6.

For each object $(A, \sigma, \tau, \phi)$ of $\mathrm{C}^{*} \operatorname{act}\left(H, K,\left(C_{0}(T), \mathrm{rt}\right)\right)$, the triple $(A, \sigma, \phi)$ is an object of $\mathrm{C}^{*} \operatorname{act}\left(H,\left(C_{0}(T), \mathrm{rt}\right)\right)$ with $H$ acting freely and properly. Thus Rieffel's theory provides an $A \rtimes_{\sigma, r} H-A^{\sigma}$ imprimitivity bimodule $Z(A, \sigma, \phi)$ which is a completion of $A_{0}:=C_{c}(T) A C_{c}(T)$. Now $\tau$ restricts to an action of $K$ on $A_{0}$ which, by the proof of [9, Proposition 4.2], extends to an action $\left(\tau \rtimes \operatorname{id}_{K}, \tau, \bar{\tau}\right)$ of $K$ on $Z(A, \sigma, \phi)$. Thus $[Z(A, \sigma, \phi), \tau]$ is an isomorphism in the category $\mathrm{C}^{*} \operatorname{act}(K)$ between $\left(A \rtimes_{\sigma, r} H, \tau \rtimes \operatorname{id}_{K}\right)=\operatorname{RCP}_{H}(A, \sigma, \tau, \phi)$ and $\left(A^{\sigma}, \bar{\tau}\right)=\operatorname{Fix}_{H}(A, \sigma, \tau, \phi)$. 
Proposition 4.2. Suppose that the action of $H$ on $T$ is free and proper. Then

$$
(A, \sigma, \tau, \phi) \mapsto[Z(A, \sigma, \phi), \tau]
$$

is a natural isomorphism between the functors $\mathrm{RCP}_{H}$ and $\mathrm{Fix}_{H}$ from the category $\mathrm{C}^{*} \operatorname{act}\left(H, K,\left(C_{0}(T), \mathrm{rt}\right)\right)$ to $\mathrm{C}^{*} \operatorname{act}(K)$.

Proof. We follow the "canonical decomposition" strategy of the proof of 6 , Theorem 3.5]. So suppose $[X, u, v]$ is a morphism from $(A, \sigma, \tau, \phi)$ to $(B, \mu, \nu, \psi)$ in $\mathrm{C}^{*} \operatorname{act}\left(H, K,\left(C_{0}(T), \mathrm{rt}\right)\right)$. Then by [6. Corollary 2.3] 2 there exists an isomorphism $[Y, u, v]:(\mathcal{K}, \zeta, \eta, \chi) \rightarrow(B, \mu, \nu, \psi)$ in $C^{*} \operatorname{act}\left(H, K,\left(C_{0}(T), \mathrm{rt}\right)\right)$ given by a $\mathcal{K}-B$ imprimitivity bimodule $Y$, and a morphism $[\kappa]:(A, \sigma, \tau, \phi) \rightarrow(\mathcal{K}, \zeta, \eta, \chi)$ in $\mathrm{C}^{*}$ act $\left(H, K,\left(C_{0}(T), \mathrm{rt}\right)\right)$ coming from a $\sigma-\zeta$ and $\tau-\eta$ equivariant nondegenerate homomorphism $\kappa: A \rightarrow M(\mathcal{K})$, such that $[X, u, v]$ is the composition:

$$
(A, \sigma, \tau, \phi) \stackrel{\kappa}{\longrightarrow}(\mathcal{K}, \zeta, \eta, \chi) \stackrel{(Y, u, v)}{\longrightarrow}(B, \mu, \nu, \psi) .
$$

Consider the following diagram in $\mathrm{C}^{*}$ act $(K)$, which is an equivariant version of diagram (4.9) from the proof of [6, Theorem 3.5]:

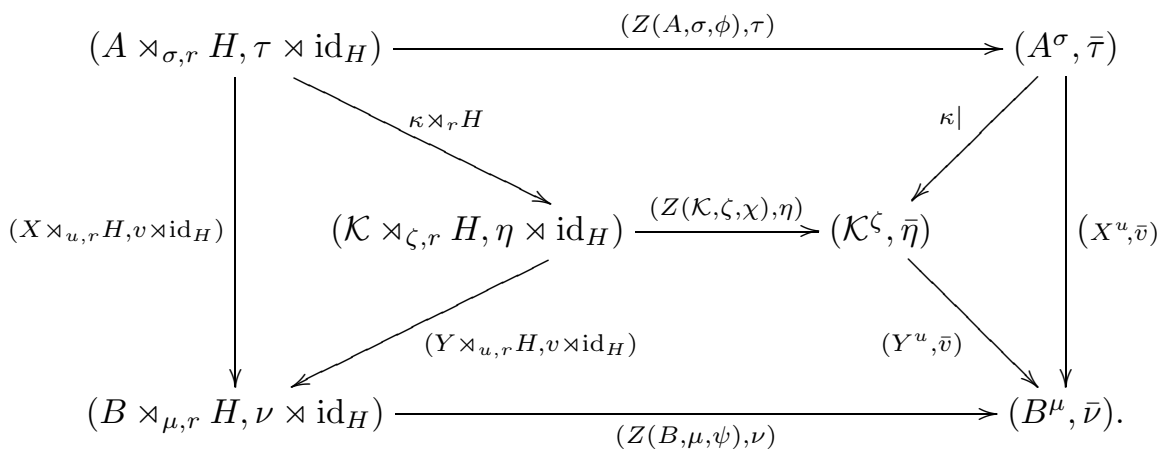

Here the arrow labeled $\kappa \rtimes_{r} H$ denotes (the isomorphism class of) $\mathcal{K} \rtimes_{\zeta, r} H$ viewed as an equivariant right-Hilbert $\left(A \rtimes_{\sigma, r} H\right)-\left(\mathcal{K} \rtimes_{\zeta, r} H\right)$-bimodule with the left action given by $\kappa \rtimes_{r} H$, and similarly for $\kappa \mid$. Thus the left and right triangles of (4.1) commute by functoriality of $\mathrm{RCP}_{H}$ and $\mathrm{Fix}_{H}$.

The upper quadrilateral of (4.1) is an equivariant version of diagram (3.1) in [10, Theorem 3.2] (see also [10, Remark 3.3]). That result provides an isomorphism $\Phi: Z(A, \sigma, \tau) \otimes_{A^{\sigma}} \mathcal{K}^{\zeta} \rightarrow Z(\mathcal{K}, \zeta, \chi)$ such that $\Phi\left(a \otimes E^{H}(c)\right)=\kappa(a) E^{H}(c)$ for $a \in A_{0}$ and $c \in \mathcal{K}_{0}=\operatorname{span}\left\{\chi(f) d \chi(g) \mid f, g \in C_{c}(T), d \in \mathcal{K}\right\}$, where $E^{H}$ is the averaging process which maps $\mathcal{K}_{0}$ into $\mathcal{K}^{\zeta}$ (see [10, Section 2]). Hence to prove that this upper quadrilateral commutes, we need to check that

$$
\Phi \circ(\tau \otimes \bar{\eta})_{k}=\eta_{k} \circ \Phi \quad \text { for } k \in K .
$$

We break off our argument for a lemma:

Lemma 4.3. For $k \in K$ and $c \in \mathcal{K}_{0}, \bar{\eta}_{k}\left(E^{H}(c)\right)=E^{H}(\eta(c))$.

\footnotetext{
${ }^{2}$ As we have observed earlier, we can apply 6] Corollary 2.3] to $\mathrm{C}^{*}$ act $\left(H \times K,\left(C_{0}(T), \mathrm{rt}\right)\right)$ without the assumption that $H \times K$ acts freely and properly on $T$.
} 
Proof. By linearity, we may suppose $c=f d g$ with $f, g \in C_{c}(T)$ and $d \in \mathcal{K}$, where to reduce clutter we write $f d g$ for $\chi(f) d \chi(g)$. Then for $h \in C_{c}(T)$ and $k \in K$, we compute, using [10, Lemma 2.2],

$$
h \bar{\eta}_{k}\left(E^{H}(f d g)\right)=\bar{\eta}_{k}\left(\left(\eta_{k}\right)^{-1}(h) E^{H}(f d g)\right)=\bar{\eta}_{k}\left(\int_{H}\left(\eta_{k}\right)^{-1}(h) \zeta_{s}(f d g) d s\right) .
$$

Thus, since the integral is norm-convergent and since $\eta$ and $\zeta$ commute, we have

$$
h \bar{\eta}_{k}\left(E^{H}(f d g)\right)=\int_{H} h \zeta_{s}\left(\eta_{k}(f d g)\right) d s=h E^{H}\left(\eta_{k}(f d g)\right) .
$$

End of the proof of Proposition 4.2, For $k \in K, a \in A_{0}$ and $c \in \mathcal{K}_{0}$, Lemma 4.3 gives

$$
\begin{aligned}
\Phi\left(\tau_{k} \otimes \bar{\eta}_{k}\left(a \otimes E^{H}(c)\right)\right) & =\Phi\left(\tau_{k}(a) \otimes \bar{\eta}_{k}\left(E^{H}(c)\right)\right) \\
& =\Phi\left(\tau_{k}(a) \otimes E^{H}\left(\eta_{k}(c)\right)\right) \\
& =\kappa\left(\tau_{k}(a)\right) E^{H}\left(\eta_{k}(c)\right) \\
& =\eta_{k}(\kappa(a)) \bar{\eta}_{k}\left(E^{H}(c)\right) \\
& =\eta_{k}\left(\kappa(a) E^{H}(c)\right) \\
& =\eta_{k}\left(\Phi\left(a \otimes E^{H}(c)\right) .\right.
\end{aligned}
$$

This establishes (4.2) and shows that the upper quadrilateral of (4.1) commutes.

Now we turn to the bottom quadrilateral of (4.1). Here, all the morphisms arise from imprimitivity bimodules, and we showed in the proof of [6. Theorem 3.5] that such a diagram commutes in $\mathrm{C}^{*}$ (that is, without the actions of $K$ ) by observing that $L(Y)^{L(u)}=L\left(Y^{u}\right)$ and that $L(Y) \rtimes_{L(u), r} H$ is isomorphic to $L\left(Y \rtimes_{u, r} H\right)$ by 8 , Proposition 3.4]. Then we applied [2, Lemma 4.6] to get the requisite imprimitivitybimodule isomorphisms. To see that the diagram commutes in $\mathrm{C}^{*}$ act $(K)$ just requires that these isomorphisms be equivariant, and this is proved in [2, Lemma 4.9]. This completes the proof of Proposition 4.2

Corollary 4.4. Suppose $H$ and $K$ act freely and properly. Then the assignment

$$
(A, \sigma, \tau, \phi) \mapsto\left[Z(A, \sigma, \phi) \rtimes_{\tau, r} K\right]
$$

is a natural isomorphism between the functors $\mathrm{RCP} \circ \mathrm{RCP}_{H}$ and $\mathrm{RCP} \circ \mathrm{Fix}_{H}$ from the category $\mathrm{C}^{*} \operatorname{act}\left(H, K,\left(C_{0}(T), \mathrm{rt}\right)\right)$ to $\mathrm{C}^{*}$.

The corollary, which can be viewed as an asymmetric version of Theorem 4.1, is an immediate consequence of Proposition 4.2 and the following elementary lemma.

Lemma 4.5. Suppose that the assignment $A \mapsto \zeta_{A}$ is a natural isomorphism between functors $F_{1}$ and $F_{2}$ into a category $C$. If $F_{3}$ is a functor defined on $C$, then the assignment $A \mapsto F_{3}\left(\zeta_{A}\right)$ is a natural isomorphism between $F_{3} \circ F_{1}$ and $F_{3} \circ F_{2}$.

Given an imprimitivity bimodule $Y$, we denote the dual bimodule by

$$
Y^{\sim}=\{b(y) \mid y \in Y\} .
$$

Proposition 4.6. Suppose $H$ and $K$ act freely and properly. For each object $(A, \sigma, \tau, \phi)$ of $\mathrm{C}^{*} \operatorname{act}\left(H, K,\left(C_{0}(T), \mathrm{rt}\right)\right)$, there is an $\left(A^{\sigma} \rtimes_{\bar{\tau}, r} K\right)-\left(A^{\tau} \rtimes_{\bar{\sigma}, r} H\right)$ imprimitivity bimodule isomorphism

$$
X(A, \sigma, \tau, \phi) \cong\left(Z(A, \sigma, \phi) \rtimes_{\tau, r} K\right)^{\sim} \otimes_{\Sigma}\left(Z(A, \tau, \phi) \rtimes_{\sigma, r} H\right),
$$


where the balanced tensor product incorporates the natural isomorphism

$$
\Sigma:\left(A \rtimes_{\sigma, r} H\right) \rtimes_{\tau \rtimes \mathrm{id}, r} K \cong\left(A \rtimes_{\tau, r} K\right) \rtimes_{\sigma \rtimes \mathrm{id}, r} H .
$$

Proof. Theorem 4.4 of [9] implies that $X(A, \sigma, \tau, \phi)$ is isomorphic to the tensor product

$$
\left(X \rtimes_{\tau, r} K\right) \otimes_{\Sigma}\left(Z(A, \tau, \phi) \rtimes_{\sigma, r} H\right),
$$

where the unfortunately named $X$ in (4.4) is a module built on $A_{0}$, as defined in the beginning of Section 2 of [9], and is equivariantly isomorphic to $Z(A, \sigma, \phi)^{\sim}$. The map on $C_{c}\left(K, A_{0}\right)$ given by $f \mapsto b\left(f^{*}\right)$, where $f^{*}(k):=\Delta_{K}(k)^{-1} \tau_{k}\left(f\left(k^{-1}\right)^{*}\right)$, extends to an imprimitivity bimodule isomorphism of $X \rtimes_{\tau, r} K$ onto $\left(Z(A, \sigma, \phi) \rtimes_{\tau, r}\right.$ $K)^{\sim}$, and the result follows.

Proof of Theorem 4.1. It follows from Corollary 4.4 that the assignment

$$
(A, \sigma, \tau, \phi) \mapsto\left[Z(A, \sigma, \phi) \rtimes_{\tau, r} K\right]^{-1}=\left[\left(Z(A, \sigma, \phi) \rtimes_{\tau, r} K\right)^{\sim}\right]
$$

is a natural isomorphism between $\mathrm{RCP} \circ \mathrm{Fix}_{H}$ and $\mathrm{RCP} \circ \mathrm{RCP}_{H}$; symmetrically,

$$
(A, \sigma, \tau, \phi) \mapsto\left[Z(A, \tau, \phi) \rtimes_{\sigma, r} H\right]
$$

is a natural isomorphism between $\mathrm{RCP} \circ \mathrm{RCP}_{K}$ and $\mathrm{RCP} \circ \mathrm{Fix}_{K}$. It is a straightforward computation to verify that the isomorphism $\Sigma$ at (4.3) indeed gives a natural isomorphism between $\mathrm{RCP} \circ \mathrm{RCP}_{H}$ and $\mathrm{RCP} \circ \mathrm{RCP}_{K}$; composing these three and using Proposition 4.6, we see that the assignment

$$
\begin{aligned}
(A, \sigma, \tau, \phi) & \mapsto\left[Z(A, \tau, \phi) \rtimes_{\sigma, r} H\right] \circ[\Sigma] \circ\left[\left(Z(A, \sigma, \phi) \rtimes_{\tau, r} K\right)^{\sim}\right] \\
& =\left[\left(Z(A, \sigma, \phi) \rtimes_{\tau, r} K\right)^{\sim} \otimes_{\Sigma}\left(Z(A, \tau, \phi) \rtimes_{\sigma, r} H\right)\right] \\
& =[X(A, \sigma, \tau, \phi)]
\end{aligned}
$$

is a natural isomorphism between $\mathrm{RCP} \circ \mathrm{Fix}_{H}$ and $\mathrm{RCP} \circ \mathrm{Fix}_{K}$, as desired.

\section{Applications}

We view objects in $\mathrm{C}^{*}$ act $(H \times K)$ as triples $(B, \alpha, \beta)$ consisting of commuting actions $\alpha: H \rightarrow$ Aut $B$ and $\beta: K \rightarrow$ Aut $B$. Suppose that the actions of $H$ and $K$ on $T$ are free and proper. Then the symmetric imprimitivity theorem of [17] says that $\operatorname{Ind}_{H}^{T}(B, \alpha) \rtimes \frac{}{\operatorname{rt} \otimes \beta} K$ is Morita equivalent to $\operatorname{Ind}_{K}^{T}(B, \beta) \rtimes \frac{}{\operatorname{rt} \otimes \alpha} H$ (and provides a concrete bimodule which is a completion of $\left.C_{c}(T, B)\right)$. As explained in [5. Corollary 3], it follows from [16, Lemma 4.1] that the reduced crossed products are Morita equivalent via a quotient $Y(B, \alpha, \beta)$ of the bimodule of [17].

Corollary 5.1. Suppose that $H$ and $K$ act freely and properly on $T$. Then the assignments

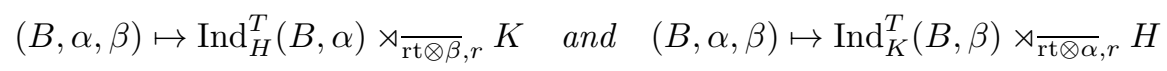

are the object maps for functors $\mathrm{F}_{H}$ and $\mathrm{F}_{K}$ on $\mathrm{C}^{*}$ act $(H \times K)$, and the assignment

$$
(B, \alpha, \beta) \mapsto[Y(B, \alpha, \beta)]
$$

is a natural isomorphism between $\mathrm{F}_{H}$ and $\mathrm{F}_{K}$. 
Proof. Define $\phi: C_{0}(T) \rightarrow M\left(C_{0}(T, B)\right)$ by $\phi(f)=f \otimes 1$. It is not hard to see that there is a functor CT : $\mathrm{C}^{*}$ act $(H \times K) \rightarrow \mathrm{C}^{*}$ act $\left(H, K,\left(C_{0}(T), \mathrm{rt}\right)\right)$ which sends the object $(B, \alpha, \beta)$ to $\left(C_{0}(T, B), \mathrm{rt} \otimes \alpha, \mathrm{rt} \otimes \beta, \phi\right)$. Then it follows from Theorem 4.1 (and general nonsense such as Lemma 4.5) that the assignment

$$
(B, \alpha, \beta) \mapsto\left[X\left(C_{0}(T, B), \mathrm{rt} \otimes \alpha, \mathrm{rt} \otimes \beta, \phi\right)\right]
$$

is a natural isomorphism between $\mathrm{RCP} \circ \mathrm{Fix}_{H} \circ \mathrm{CT}$ and $\mathrm{RCP} \circ \mathrm{Fix}_{K} \circ \mathrm{CT}$. Therefore we can complete the proof of the corollary by showing that the relevant generalized fixed-point algebras coincide with the induced algebras, and that the modules $Y(B, \alpha, \beta)$ and $X\left(C_{0}(T, B), \mathrm{rt} \otimes \alpha, \mathrm{rt} \otimes \beta, \phi\right)$ are isomorphic as imprimitivity bimodules, hence define the same isomorphism in the category $\mathrm{C}^{*}$.

The dense subalgebra $A_{0}=C_{c}(T) C_{0}(T, B) C_{c}(T)$ of $C_{0}(T, B)$ is $C_{c}(T, B)$. Then for $f, g \in A_{0}$, the $\operatorname{Fix}\left(C_{0}(T, B), \operatorname{rt} \otimes \alpha\right)$-valued inner product $\langle f, g\rangle$ is multiplication by the function

$$
s \mapsto \int_{H} \alpha_{t}\left(f(s t)^{*} g(s t)\right) d t,
$$

which is the same as the $\operatorname{Ind}_{H}^{T}(B, \alpha)$-valued inner product on $C_{c}(T, B)$ in [17]. Hence $\operatorname{Fix}\left(C_{0}(T, B), \mathrm{rt} \otimes \alpha\right)$ and $\operatorname{Ind}_{H}^{T}(B, \alpha)$ are the same subalgebra of $M\left(C_{0}(T, B)\right)$. A similar argument applies to $\operatorname{Ind}_{K}^{T}(B, \beta)$.

To see that $Y(B, \alpha, \beta)$ is isomorphic to $X\left(C_{0}(T, B), \mathrm{rt} \otimes \alpha, \mathrm{rt} \otimes \beta, \phi\right)$, we just need to check that the two sets of actions and inner products on $C_{c}(T, B)$ coincide, and this is a straightforward calculation.

When $K=\{e\} \operatorname{Ind}_{K}^{T}(B, \beta)=C_{0}(T, B)$ and [5, Corollary 4] implies that

$$
C_{0}(T, B) \rtimes_{\mathrm{rt} \otimes \alpha} H=C_{0}(T, B) \rtimes_{\mathrm{rt} \otimes \alpha, r} H .
$$

Both the bimodule $X\left(C_{0}(T, B), \mathrm{rt} \otimes \alpha, \mathrm{id}, \phi\right)$ and the bimodule $W(B, \alpha)$ in 18 are completions of $C_{c}(T, B)$, and again the formulas for the actions and inner products turn out to be the same. Hence we obtain the naturality of the Morita equivalence for diagonal actions from [18].

Corollary 5.2. Suppose that $H$ acts freely and properly on $T$. Then there are functors on $\mathrm{C}^{*} \operatorname{act}(H)$ whose maps on objects are given by

$$
(B, \alpha) \mapsto \operatorname{Ind}(C, \alpha) \quad \text { and } \quad(B, \alpha) \mapsto C_{0}(T, B) \rtimes_{\mathrm{rt} \otimes \alpha} H,
$$

and the assignment

$$
(B, \alpha) \mapsto[W(B, \alpha)]
$$

is a natural isomorphism between these two functors.

\section{REFERENCES}

[1] J. Chabert, S. Echterhoff and H. Oyono-Oyono, Shapiro's lemma for topological K-theory of groups, Comment. Math. Helv. 78 (2003), 203-225. MR1966758 (2004c:19005)

[2] S. Echterhoff, S. Kaliszewski, J. Quigg and I. Raeburn, A categorical approach to imprimitivity theorems for $C^{*}$-dynamical systems, Mem. Amer. Math. Soc. 180 (2006), no. 850, viii+169 pages. MR 2203930(2007m:46107)

[3] R. Exel, Morita-Rieffel equivalence and spectral theory for integrable automorphism groups of $C^{*}$-algebras, J. Funct. Anal. 172 (2000), 404-465. MR.1753180 (2001h:46104)

[4] P. Green, $C^{*}$-algebras of transformation groups with smooth orbit space, Pacific J. Math. 72 (1977), 71-97. MR0453917 (56:12170)

[5] A. an Huef and I. Raeburn, Regularity of induced representations and a theorem of Quigg and Spielberg, Math. Proc. Camb. Phil. Soc. 133 (2002), 249-259. MR.1912400(2003g:46080) 
[6] A. an Huef, S. Kaliszewski, I. Raeburn and D.P. Williams, Naturality of Rieffel's Morita equivalence for proper actions, Algebr. Represent. Theory 14 (2011), 515-543. MR2785921

[7] A. an Huef, S. Kaliszewski, I. Raeburn and D.P. Williams, Fixed-point algebras for proper actions and crossed products by homogeneous spaces, to appear in Illinois J. Math. (arXiv:0907.0681)

[8] A. an Huef, I. Raeburn and D.P. Williams, Proper actions on imprimitivity bimodules and decompositions of Morita equivalences, J. Funct. Anal. 200 (2003), 401-428. MR.1979017 (2004j:46089)

[9] A. an Huef, I. Raeburn and D.P. Williams, A symmetric imprimitivity theorem for commuting proper actions, Canad. J. Math. 57 (2005), 983-1011. MR2164592 (2006f:46067)

[10] S. Kaliszewski, J. Quigg and I. Raeburn, Proper actions, fixed-point algebras and naturality in nonabelian duality, J. Funct. Anal. 254 (2008), 2949-2968. MR2418615 (2010a:46160)

[11] G.G. Kasparov, Equivariant KK-theory and the Novikov conjecture, Invent. Math. 91 (1988), 147-201. MR918241 (88j:58123)

[12] E. Kirchberg and S. Wassermann, Permanence properties of $C^{*}$-exact groups, Doc. Math. 4 (1999), 513-558. MR.1725812 (2001i:46089)

[13] P.S. Muhly, J. Renault and D.P. Williams, Equivalence and isomorphism for groupoid $C^{*}$ algebras, J. Operator Theory 17 (1987), 3-22. MR873460 (88h:46123)

[14] P.S. Muhly and D.P. Williams, Equivalence and disintegration theorems for Fell bundles and their $C^{*}$-algebras, Dissertationes Math. 456 (2008), 1-57. MR2446021 (2010b:46146)

[15] D. Pask and I. Raeburn, Symmetric imprimitivity theorems for graph $C^{*}$-algebras, Internat. J. Math. 12 (2001), 609-623. MR1843869 (2002g:46114)

[16] J.C. Quigg and J. Spielberg, Regularity and hyporegularity in $C^{*}$-dynamical systems, Houston J. Math. 18 (1992), 139-152. MR1159445 (93c:46122)

[17] I. Raeburn, Induced $C^{*}$-algebras and a symmetric imprimitivity theorem, Math. Ann. 280 (1988), 369-387. MR936317 (90k:46144)

[18] I. Raeburn and D.P. Williams, Pull-backs of $C^{*}$-algebras and crossed products by certain diagonal actions, Trans. Amer. Math. Soc. 287 (1985), 755-777. MR768739 (86m:46054)

[19] J. Renault, Représentation des produits croisés d'algèbres de groupoïdes, J. Operator Theory 18 (1987), 67-97. MR.912813 (89g:46108)

[20] M.A. Rieffel, Applications of strong Morita equivalence to transformation group $C^{*}$-algebras, Operator Algebras and Applications, Proc. Symp. Pure Math., vol. 38, Part I, Amer. Math. Soc., Providence, RI, 1982, pages 299-310. MR679709 (84k:46046)

[21] M.A. Rieffel, Proper actions of groups on $C^{*}$-algebras, Mappings of Operator Algebras, Progress in Math., vol. 84, Birkhäuser, Boston, 1990, pages 141-182. MR.1103376 (92i:46079)

[22] M.A. Rieffel, Integrable and proper actions on $C^{*}$-algebras, and square-integrable representations of groups, Expositiones Math. 22 (2004), 1-53. MR2166968 (2006g:46108)

Department of Mathematics and Statistics, University of Otago, P.O. Box 56, DuneDIN 9054, NEW ZEALAND

E-mail address: astrid@maths.otago.ac.nz

School of Mathematical and Statistical Sciences, Arizona State University, Tempe, ARIZONA 85287-1804

E-mail address: kaliszewski@asu.edu

Department of Mathematics and Statistics, University of Otago, P.O. Box 56, DuneDIN 9054, NEW ZEALAND

E-mail address: iraeburn@maths.otago.ac.nz

Department of Mathematics, Dartmouth College, Hanover, New Hampshire 03755

E-mail address: dana.williams@dartmouth.edu 